\title{
Multiple Prime Expansion Channel Hopping for Blind Rendezvous in a Wireless Sensor Network
}

\author{
Zhou Zhixin $\mathbb{D}^{1},{ }^{1}$ Yanjun Deng $\mathbb{D},{ }^{1}$ Zhang Xiaohong $\mathbb{D}^{1},{ }^{1}$ Zhang Xianfei $\mathbb{D},{ }^{1}$ Hu Liqin $\mathbb{D},{ }^{2}$ \\ and Zhao Zhidong $\mathbb{D}^{3}$ \\ ${ }^{1}$ School of Electronic Information, Hangzhou Dianzi University, Zhejiang, Hangzhou 310035, China \\ ${ }^{2}$ Zhejiang College of Construction, Hangzhou, China \\ ${ }^{3}$ School of Cyberspace, Hangzhou Dianzi University, Zhejiang, Hangzhou 310035, China
}

Correspondence should be addressed to Zhao Zhidong; zhaozd@hdu.edu.cn

Received 24 November 2021; Accepted 15 January 2022; Published 9 February 2022

Academic Editor: Liqin Shi

Copyright ( 2022 Zhou Zhixin et al. This is an open access article distributed under the Creative Commons Attribution License, which permits unrestricted use, distribution, and reproduction in any medium, provided the original work is properly cited.

A channel rendezvous is a significant aspect of communication. In this context, blind rendezvous is the process of selecting a common available channel and establishing a communication link for wireless devices in a wireless sensor network. The rendezvous of asymmetric and heterogeneous wireless devices is a challenge. Thus, to improve speeds and stability of rendezvous, we analyze time slot overlap and channel determinism in the rendezvous algorithm and propose a rendezvous algorithm named Multiple Prime Expansion (MPE). In a final simulation study, we compare the performance of the MPE with other existing algorithms in an asymmetric and heterogeneous scenario. Results show that MPE has excellent performance for the ATTR and MTTR.

\section{Introduction}

In recent years, numerous cities in China have built an Internet of Things projects to improve the mobility and carrying capacity of data in public fields. These projects can boost the speed of data collection and optimize city management and services [1]. Rail transportation services use data to adjust station populations and smartphone applications to improve the efficiency of targeted services based on user access data [2]. At the same time, cities protect the supply of water, food, and energy through billing data [3-6].

Currently, more and more data collection systems are based on Wireless Sensor Networks (WSNs) [7]. Thus, compared to wired transmission systems, WSN enhances flexibility but requires more resilient energy and timeliness cost. Energy consumption and data timeliness have important implications for WSNs.

Routing protocols are widely used in wireless surveillance as tools that can organize wireless nodes to collect information in an orderly manner [8]. A number of researchers have improved routing protocols to reduce wireless monitoring energy consumption. These are divided into several groups, such as node deployment, clustering techniques, and transmission methods $[9,10]$. This method can be applied for both applications and engineering [11].

The connection speed of sensors is an important variable in data timeliness research. The rapid increase in sensor integration [12] and data demand greatly enhanced the demand for the number of wireless devices in WSN and hence wireless spectrum. This has led to a dramatic increase in demand for wireless spectra and so this has become a scarce resource. However, spectrum scarcity and low spectrum utilization persist in rendezvous for wireless devices has proven challenging in resource-scarce settings [13]. Thus, to achieve efficient data transmission among sensors, communicating parties must adopt an efficient and robust mechanism to complete data interaction. Blind channel rendezvous, without a common control channel (CCC), has garnered a growing interest [14]. Channel hopping is a typical technique used in most blind channel rendezvous. Wireless devices access the channels in different time slots according to a predefined frequency hopping sequence, and 
rendezvous is successfully achieved when the two users access the same common available channel in a certain time slot.

Time to rendezvous (TTR) is also a key factor. However, early in development, some devices initially make random sequences to blind rendezvous, which would make rendezvous time unpredictable. Therefore, during the operation of the wireless device, we needed to identify a precondition that contained enough frequency of rendezvous for data transmission. Wireless devices needed to exchange data multiple times during operation, which made minimizing TTR a direction in research. For researchers, any channels between users can directly be used as a sequence without any preprocessing. In other words, the effectiveness of the channel sequence directly affects the stability of rendezvous. Details of the technical specifications and requirements for the algorithm are provided as follows.

(1) Degree of rendezvous (DR): This can be defined by dividing the number of channels that have completed rendezvous by the total number of channels. Thus, when $\mathrm{DR}=1$, this is referred to as complete rendezvous. This means that all channels in the sequence can be rendezvous, and high DR can avoid channel blocking and improve channel utilization.

(2) Frequency of rendezvous (FR): The number of rendezvous times divided by the frequency hopping slot is FR. FR describes the strength of rendezvous across the whole frequency hopping cycle.

(3) Average TTR (ATTR): ATTR refers to the average time between two consecutive rendezvous in the frequency hopping cycle, which is the average value of the rendezvous time. ATTR describes the speed of the rendezvous.

(4) Maximum TTR (MTTR): The longest time period from start to rendezvous is MTTR. Thus, if the sequence cannot guarantee rendezvous, MTTR is infinite. In other words, as the length of the hopping sequence increases, the MTTR is increasing obviously.

Our current approach meets these four above requirements and suggests interesting directions for research and applications. According to starting time, the hopping sequences can be classified as synchronous sequences and asynchronous sequences. Lin et al. proposed a multiradio channel-hopping scheme (CHS) that preserves network connectivity and synchronous sequences [15]. Another algorithm, ASCH (asymmetric synchronous channel hopping), divides the whole sets of channels into several levels to meet asynchronous sequences [16]. However, in most cases, the wireless devices have local clocks that make it difficult to achieve synchronization. Some asynchronous algorithms have been proposed, such as in the work of Liu, who considered the impact of network factors (channel availability and multiuser contention) when designing the frequency hopping sequence [17]. Wang proposed MAAPS based on rendezvous-success rate and variance [18].
Hopping sequences can be divided into homogeneous sequences and heterogeneous sequences based on different available channels $[19,20]$. A homogeneous model includes symmetric/asymmetric channel sets such as GOS [21] and CRESQ [22].

We, therefore, investigate the two requirements in WSN for the rendezvous algorithm. On the one hand, there are various sensors with different monitoring data types and frequencies in WSN [23], which means that the available channels are heterogeneous. On the other, as data fusion is an essential requirement for WSN, hopping sequences are required to meet the asymmetric condition. Therefore, the nonpreprocessing rendezvous algorithm used in WSN needs to satisfy symmetric and asymmetric requirements on a heterogeneous basis.

The above analysis suggests that the investigation of the rendezvous algorithm is essential. Only a little information is currently available about the asymmetric and heterogeneous rendezvous algorithm of WSN. In this work, we propose a MPE (Multiple Prime Expansion) algorithm to match asymmetric and heterogeneous requirements. The MPE examined the feedback relationship between the length of a sequence and TTR to strengthen the discrimination subsequences. The rendezvous algorithm switches to another operation mode when a subsequence reaches certain conditions.

\section{Problem Statement}

The time slot communication system was measured using unit time, which includes time slots, channels, and frequency hopping rules. The slot in slot communication system corresponds to a minimum time interval to select a communication link in the communication network. Another study focused on channel frequency hopping sequence, which can be regarded as a specific access sequence for a rendezvous algorithm. In each process, wireless devices access the designated channel by channel frequency hopping sequence to establish contact with other devices on each channel. In this study, we introduce several difficulties relating to channel hopping technology in WSN.

2.1. Limitations of WSN Conditions. Wireless sensors were analyzed with channel information connected to other wireless devices via antennae and matched with local channels. There are, however, some difficulties in using the rendezvous algorithm in WSN compared with other fields. These existing rendezvous algorithms are not yet efficient enough to be used in WSN due to special constraints. The main constraints on the frequency hopping sequences are shown to be as follows.

(1) The rendezvous rules cannot be easily changed during frequency hopping, where WSNs are usually incorporated into a system together with servers, mobile applications, and databases [24].

(2) According to its different functions, different sensor matching schemes must be designed. In detail, WSNs contain various types of sensors, like power, 
telecommunications, water supply, and natural gas sensors. It causes a heterogeneous relationship between wireless devices. Thus, symmetric and asymmetric requirements need to be considered in rendezvous algorithm design.

(3) Distinct working frequencies develop at various times under different types of sensors. Thus, symmetric and asymmetric requirements need to be considered in the rendezvous algorithm.

2.2. Problem Formulation. We assume that there are two wireless devices to rendezvous. In our sequence, each device is equipped with one antenna, which only tries to rendezvous with one device in a time slot. Thus, let $S_{1}(i)$ and $S_{2}(i)$ denote the sequences of two wireless devices in the rendezvous algorithm and let $j \in\{1,2, \ldots, j, \ldots, S\}$ denote the rendezvous time from slot 1 to slot $S$, where $S$ denotes the total number of slots. When the rendezvous occurs at slot $j$.

This means that the rendezvous problem in WSN can be formulated as follows:

$$
\min _{j}\left(\sum_{i=1}^{j} \operatorname{sign}\left(\left|S_{1}(i)\right|-\left|S_{2}(i)\right|\right)\right) .
$$

\section{Materials and Methods}

We assessed the challenges above using the improved algorithm MPE as detailed below.

As WSN technology use has increased, more and more research has focused on the timeliness of data exchange. The use of the rendezvous algorithm is key to this task. Two issues need to be considered, however, when designing a rendezvous algorithm: (A) rendezvous within a limited time and (B) a reduction in rendezvous time.

The MPE rendezvous algorithm enables the inevitability of the rendezvous and reduces rendezvous time which enables rapid data exchange for the WSN. The algorithm consists of five phases: (a) given global channels; (b) any two wireless devices have the possibility of rendezvous (common channel); (c) achieve inevitability of rendezvous; (d) create subsequences; (e) subsequence is expanded into a frequency hopping sequence.

3.1. Network Model. We have made some assumptions based on the above constraints caused by WSN.

(1) We assume multiple nonoverlapping channels and each wireless device can sense a part of the channel.

(2) We allow each wireless device to be equipped with only one cognitive radio transceiver (antenna), which can sense the channel status and switch to different channels without auxiliary means.

(3) All wireless devices are anonymous in the network (without ID).

(4) Each wireless device has a local clock.
(5) The sensing device includes a network mapping function. Different wireless terminals use a channel of a unified set of channel indexes. However, the common mapping function is outside the scope of this paper.

(6) The length of each slot is the same.

(7) We allow $M$ wireless devices. Thus, if we let $M_{u}\left(M_{u} \subseteq M, M_{u} \neq \varnothing\right)$ denote the available channel set of wireless devices $u$. For any two wireless devices $u$ and $v$, their locally available channel sets $C_{u}$ and $C_{v}$ may have different channels. In order to ensure the rendezvous, we assume that any two nodes have the possibility of rendezvous. That is, there is at least one publicly available channel $\left(\forall u, v \in M, C_{u} \cap C_{v} \neq \varnothing\right)$.

3.2. Slot Symmetry. Channel hopping sequences are usually periodic. The smallest repeating unit of the channel hopping sequence is called the subsequence. As the rule for the construction of the channel hopping sequence is fixed, the structure in each subsequence has been restricted. The intersection of any two sequences shows periodicity with the repetition of the subsequence. Therefore, the rendezvous algorithm is the generation and connection method of the subsequence with rendezvous ability.

According to the start time of channel hopping sequences, the channel hopping sequences were divided into synchronous sequences and asynchronous sequences, as shown in Figure 1.

An asynchronous channel hopping sequence presents two different asynchronous situations, as shown in Figures 2(a) and 2(b).

The asynchronous time slot communication system is shown in Figure 2. The time slots in Figure 2(a) are $P$, and the two sequences are separated by $T 2$ time slots. The time slots in Figure 2(b) are $Q$, and the gap of the start time of the two sequences in the figure is $(T 2+k)$ time slots.

It is clear that in Figure 2(b), T2 is a nonnegative integer, $k \in[0,1]$, the time slots of these two sequences are not aligned. As there is no time slot alignment information, the wireless devices decide the moment when the wireless devices start to rendezvous. In other words, the rendezvous of the asynchronous time slot system is more complicated. Therefore, the asynchronous nonaligned time slot communication system can be transformed into an aligned time slot communication system.

As the communication system of the asynchronous time slot satisfies $Q=2 P$, an asymmetric asynchronous time slot can be considered a symmetric slot communication system.

We assume that the minimum time interval required for any two wireless devices to establish a communication link is $Q$ time slots. In an asynchronous communication system, the overlap range of rendezvous time slots can be divided into the following two situations.

Situation 1: the range of $k$ is $[0, Q / 2)$, as shown in Figure 3: 


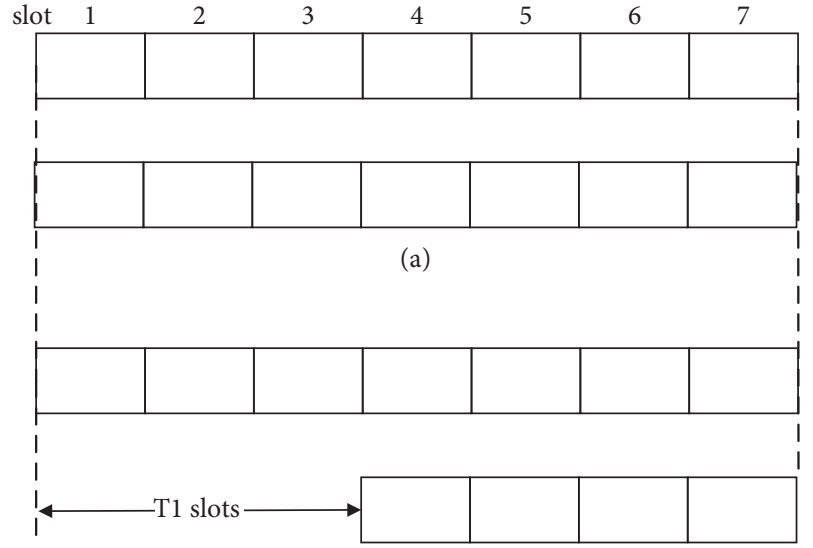

(b)

FIGURE 1: Synchronous/asynchronous channel hopping sequences.

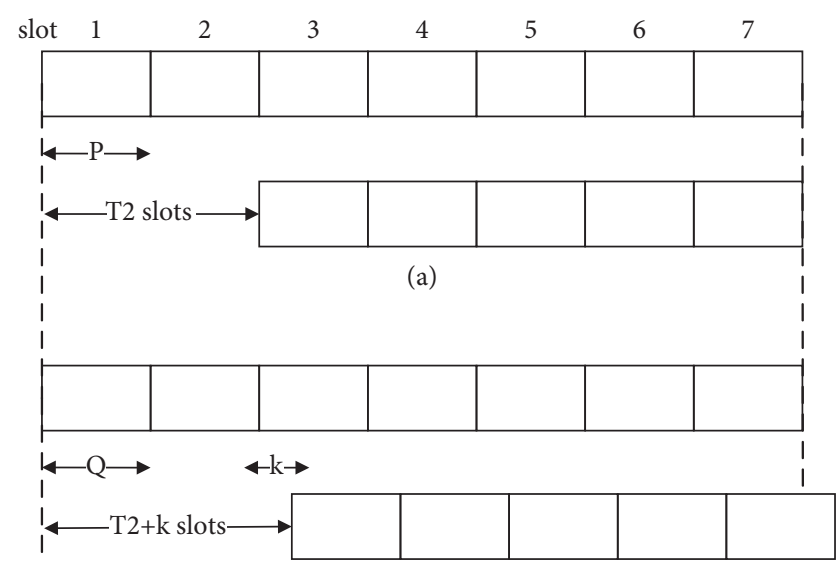

(b)

Figure 2: Asynchronous aligned/nonaligned time slots.

$$
Q-k=2 P-k \geq P .
$$

The length of the time slot shown in red in Figure 3 can satisfy the asynchronous channel hopping sequences to complete rendezvous.

Situation 2: The range of $k$ is $[0, Q / 2)$, as shown in Figure 4:

$$
Q-(2 P-k)=k \geq P .
$$

The length of the time slot shown in red in Figure 4 also can allow asynchronous channel hopping sequences to complete rendezvous.

Section 3.2 verifies that the asymmetric time slot communication sequence can be regarded as the symmetric channel hopping sequence for rendezvous. In other words, when time slots in the network all have the same length, the time required to establish a communication link is at least two time slots.

3.3. Algorithm Processes. As mentioned in Section 2, the channel frequency hopping sequence is limited by the heterogeneity of wireless devices. Otherwise, it will waste a lot of time that uses the traditional rendezvous algorithm. As there are some differences between the available channels of wireless devices and the total number of channels. It is, therefore, crucial to design a suitable rendezvous algorithm for asymmetric channel environments. The algorithm model is shown in Figure 5.

The MPE algorithm needs to initially rendezvous in a limited time. Since the number of available channels for each wireless device is different, the channel frequency hopping sequence of the MPE algorithm is unequal in different devices. We assume that $D$ and $E$ ( $D$ and $E$ are both periodic sequences), respectively, represent the channel sequences of two wireless devices. $D$ is composed of $d$ cycle of subsequence, and $E$ is composed of the base sequence $e$ cycle. $x$ and $y$ are coprime numbers, and both $x$ and $y$ are not less than the number of available channels, and $c$ is the total number of channels. The verification of the inevitability of rendezvous in MPE is as follows.

$D=\left\{d_{1}, d_{2}, d_{3}, \ldots, d_{y}\right\}$ means the sequence containing $y$ repeating subsequences $d=\left\{c_{1}, c_{2}, c_{3}, \ldots, c_{x}\right\}$. $E=\left\{e_{1}, e_{2}, e_{3}, \ldots, e_{y}\right\}$ means the sequence containing $y$ repeating subsequences $e=\left\{c_{1}, c_{2}, c_{3}, \ldots, c_{y}\right\}$.

Assume that any element $c$ in $d$ ( $d$ is a subsequence of $D$ ) corresponds to $c_{t}\left(c_{t} \in E\right)$. Then in the $m$ th and $n$th subsequences, $c$ corresponds to $c(t+m x) \% y$ and $c(t+n x) \% y$ in $E$, respectively. Thus, if

$$
c(t+m x) \% y=c(t+n x) \% y,
$$

then $[(m-n) x] \% y=0$ can be derived. That is, $(m-n) x / y=k, k$ is a natural number.

As $x$ and $y$ are prime numbers and $x \neq y x \neq y$, their relationship is elucidated by formula (5), as follows:

$$
m-n=q y(q=1,2,3, \ldots)
$$

This can be established by calculating $(m-n) x / y=k$ only when $k=x$. Therefore, in the following $y$-1 cycles, $\forall c \in d$ will correspond to $y$ different elements in E. If $\exists c \in D, c \in E$, the sequences $D$ and $E$ will inevitably meet within $y$ cycles, and, therefore, $q$ is a natural number.

Thus, if we assume that subsequences of $D$ and $E$ have common channels and the lengths are different prime numbers, there are rendezvous between $D$ and $E$. When $x$ and $y$ are different prime numbers, there is $\forall c_{i} \in d$ corresponding to all elements in $e$. This means that sequences $D$ and $E$ will achieve rendezvous during a finite time.

Subsequent to research into rendezvous inevitability, more recently, the focus has shifted to reducing rendezvous time between two wireless devices. Since the rendezvous time depends on the channel size (number of channels), the aspect of reducing the rendezvous time based on global channels was the focus of the present work.

Assuming that the number of global channels is $M$. There are two wireless devices waiting to rendezvous in the network. Each wireless device only contained one antenna, and each antenna has only accessed a channel in a time slot. The available channels of the two wireless devices all belong to global channels. Let A and B be the number of available channels of the two wireless devices, respectively. In order to 


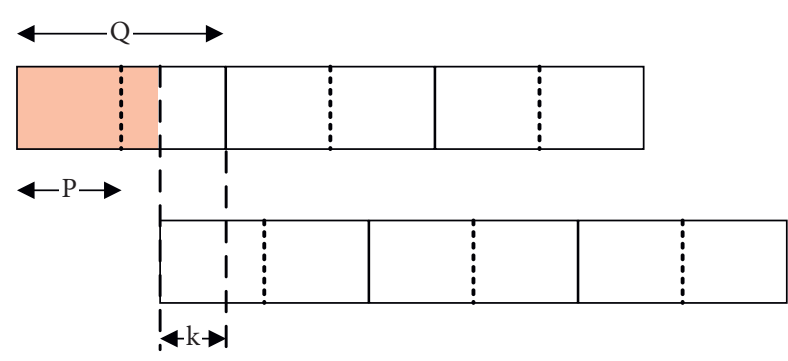

Figure 3: Asynchronous nonaligned-situation 1.

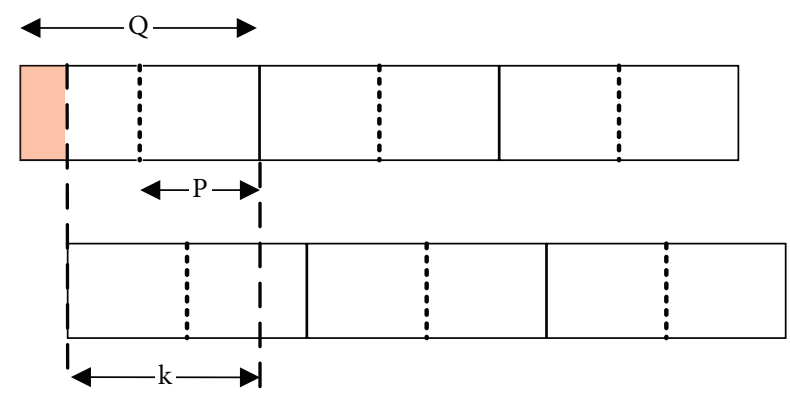

FIGURE 4: Asynchronous nonaligned-situation 2.

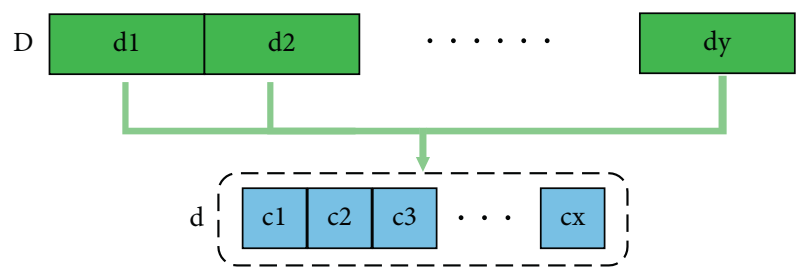

subsequence

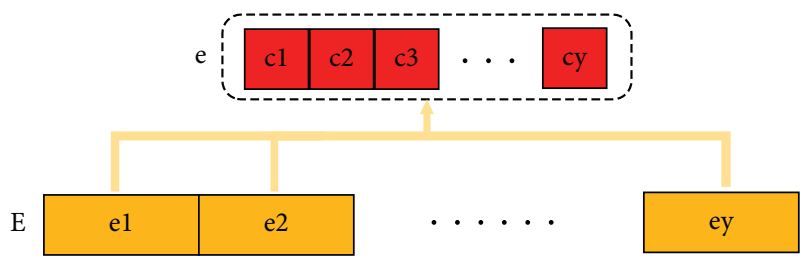

FIGURE 5: Common channel diagram.

achieve rendezvous, it is necessary to have at least one common channel between two wireless devices, as shown in Figure 6.

According to Section 3.3, when the length of the subsequences is different prime, the two sequences can achieve rendezvous in a limited time. Therefore, $\mathrm{A}$ and $\mathrm{B}$ need to be expanded into LA and LB with prime lengths. That is to say, both LA and LB are composed of two parts, the "original part" (A or B) and the "fill part" (FA or FB). The goal of the present work was to increase the probability of the common channel in the "fill part"(FA or FB). Different fill part conditions are caused by different lengths of original parts. Different methods to design "fill part" can be caused by different lengths of "original parts." The "fill part" selects at least one channel number from the original channel (A or $\mathrm{B})$. Then when the "original part" is expanded into a subsequence with a prime number, the probability that a single subsequence contains a common channel can be increased. Two cases are considered in the following research to design the "fill part."

In the first case, when $A \neq B$. We let $p$ denotes the probability that the "original part" contains common channels, and $m$ denote the length of the "fill part". The "fill part" is generated from the "original part." The "fill part" contains the probability $p_{A \neq B}$ of the common channels, and the calculation process is shown in the following formula:

$$
p_{A \neq B}=\frac{1}{2^{m}} \sum_{i=0}^{m} p C_{m}^{i}(1-p) C_{m}^{m-i} .
$$

In the second case, when $A=B$, formula (7) has suggested there is more overlap part between $\mathrm{A}$ and $\mathrm{B}$ than in the previous case. In other words, the second case tends to have more common channels. We can fill in the "fill part" by random nonrepeated channels from the "original part." The "fill part" contains the probability $p A=B$ of the common channels, and the calculation process is shown in the following formula:

$$
p_{A=B}=\frac{m p}{L_{A}}
$$

The designing process of MPE algorithm is presented in Table 1.

\section{Performance Evaluation}

4.1. Simulation Environment. In this section, we used simulation experiments to evaluate the performance of the proposed algorithm and verify the above assumptions. The parameters are shown in Table 2. MATLAB was used to simulate the rendezvous process. The time for a successful rendezvous is influenced, as mentioned earlier, by the number of global channels and the distribution of common channels. Therefore, the experiment mainly aimed to simulate both symmetric and asymmetric rendezvous algorithms under heterogeneous conditions. The entire simulation process was repeated 2000 times, and MTTR and ATTR over all the simulations were recorded.

4.2. Experimental Results and Analysis. This section simulates the MPE algorithm in the symmetrical and asymmetrical channel scenarios. The performance evaluation indicators are the longest convergence time and the average convergence time. When in a symmetrical/asymmetric situation, this article uses the MPE algorithm with JS (JumpStay) [25], ACH (asynchronous channel hopping rendezvous algorithm) [26], RW (receiver wait for rendezvous algorithm) [27], AHW (alternate hop-and-wait channel rendezvous algorithm), and SSB (short sequence-based rendezvous algorithm) [28] for comparison.

Figure 7 is the comparison result of MTTR in a symmetrical scenario. An upward trend of MTTR could be observed in all algorithms with the global channels increasing. Since the MTTR of CRSEQ is much higher than that of other algorithms, only a part of the data is shown in 


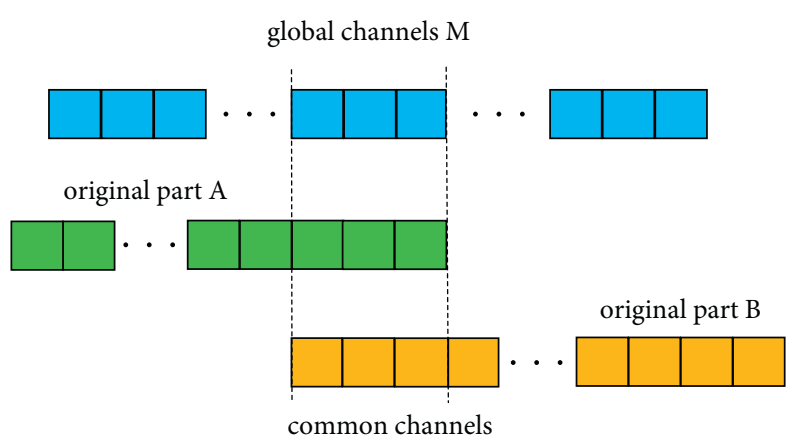

Figure 6: Common channel diagram.

TABLE 1: Multiple prime expansion algorithm.

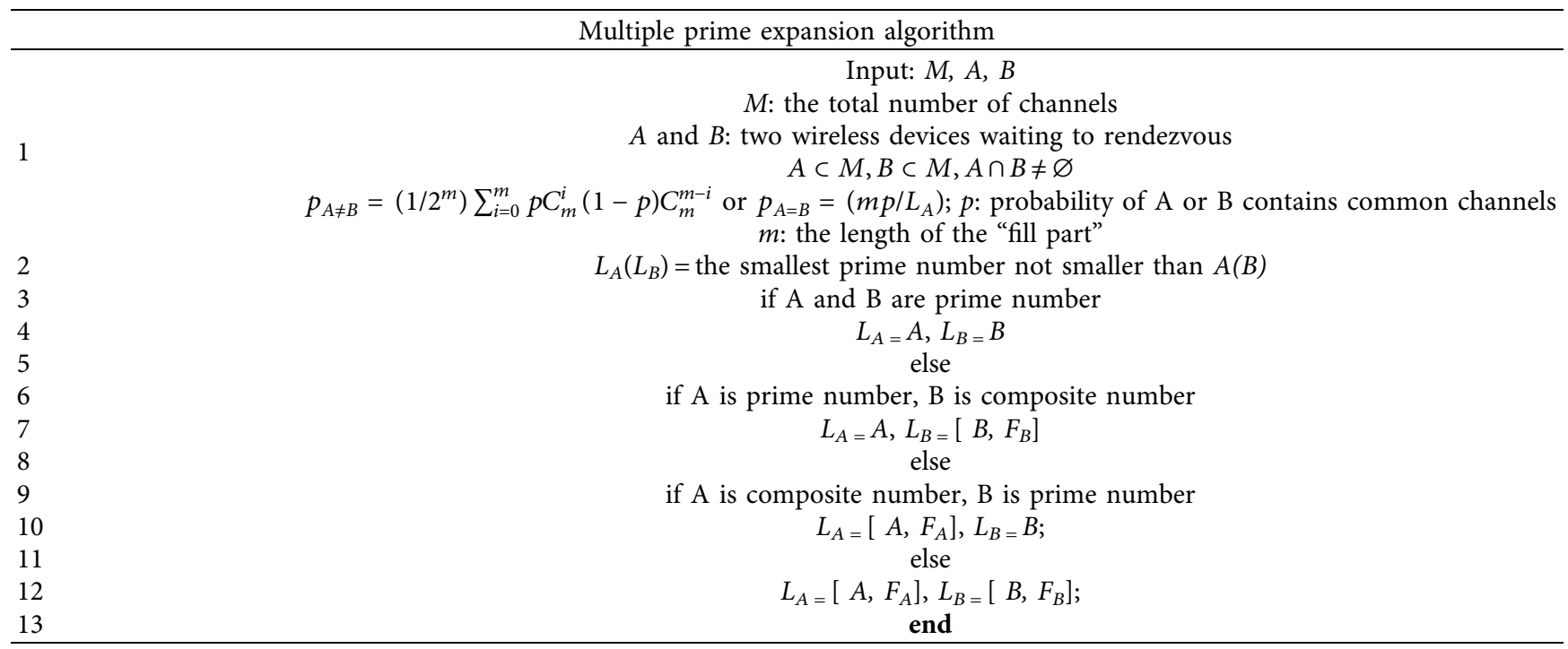

TABLE 2: Parameter setting of simulating experiments.

\begin{tabular}{lc}
\hline Simulating parameter setting & Value \\
\hline Number of channels & $5 \sim 50$ \\
Experiment time & 2000 \\
Number of wireless devices & 2 \\
Analysis type & heterogeneous/symmetrical \\
\hline
\end{tabular}

Figure 7. As for MPE, although the length of the sequence of the MPE algorithm is slightly larger than other algorithms, the common channel ratio is increased in "fill part" in MPE, and thus rendezvous can be completed faster for MPE. In symmetrical instances, when there are fewer global channels, MPE's MTTR is close to RW and ACH. One likely reason for this experimental result is that the "fill part" of MPE does not effectively improve the probability of common channels. The MPE algorithm is better than other algorithms in MTTR when there are many common channels between wireless devices.

Figure 8 is a comparison result of ATTR in the symmetrical scenario. In the experiment, the ATTR of each algorithm has more obvious fluctuations compared with MTTR. Among them, after the number of global channels of CRSEQ exceeds 20, ATTR increases rapidly, which substantially limited rendezvous multiple times in a limited time. As shown in Figure 8, the common channels show an upward trend as global channel scale expansion, which makes the MPE algorithm show better rendezvous stability. Compared with SSB, the ATTR of the MPE algorithm is much smaller than the SSB algorithm, with an average decrease of $63.9 \%$. In the entire simulation process, the MPE algorithm also achieves the shortest ATTR. Compared with the $\mathrm{ACH}$ and RW algorithms, respectively, the average decrease is $71.55 \%$ and $87.43 \%$. This shows that the MPE algorithm has a strong continuous capability to rendezvous.

Figure 9 is the comparison result of MTTR in the asymmetric scenario. Compared with the symmetric result, there are two obvious characteristics. (1) The MTTR under asymmetric conditions shows a nonlinear upward trend. (2) In the symmetric scenario, there appears to be no obvious gap of MTTR between most rendezvous algorithms. However, in the asymmetric scenario, the rendezvous certainty of each algorithm will be affected by the symmetry scenario. The difficulty will be significantly increased with the fast loss of symmetry.

From Figure 9, we can see that the MTTR of JS and RW is much higher than the other algorithms (MTTR >10000) with an acceleration trend. The MTTR of MPE is very close 


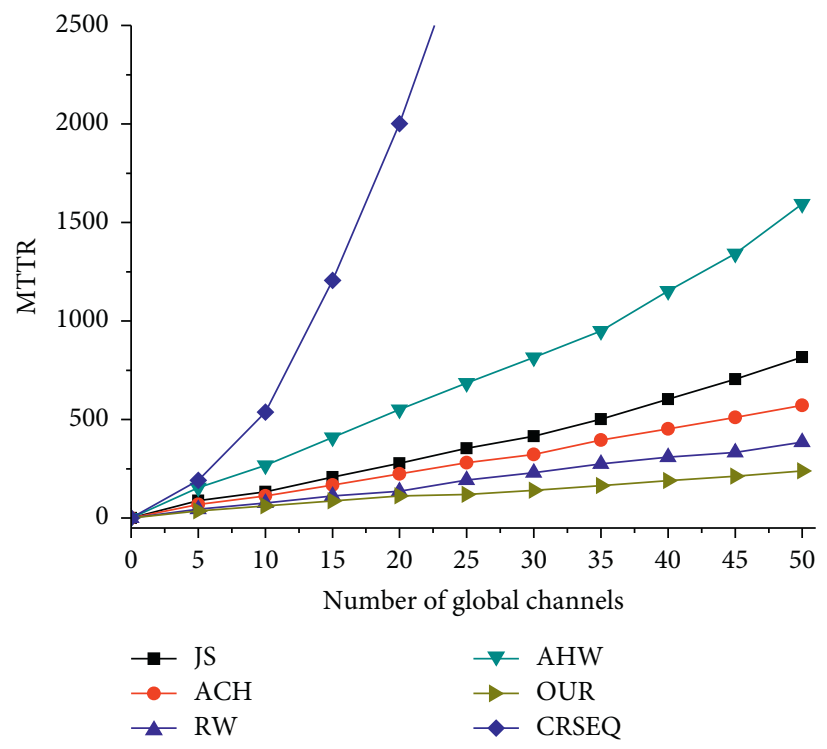

FIgURE 7: MTTR in the symmetrical scenario.

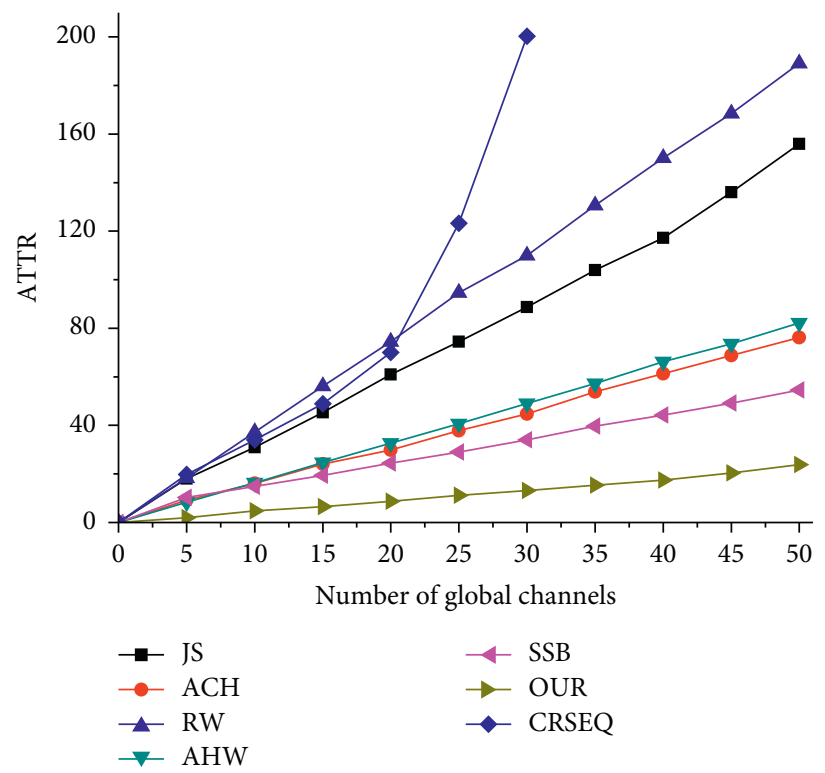

FIgURE 8: ATTR in a symmetrical scenario.

to AHW when global channels have a smaller scale. However, after the number of channels is more than 30 , the MTTR of the MPE algorithm keeps steady. In contrast, all other algorithms showed varying degrees of rapid rise. For example, MTTR $(M=50)$ compared with MTTR $(M=25)$, AHW increased by $236.88 \%$, and the average growth rate of SSB (every 5 channels) is as high as $59.3 \%$. The MTTR of the MPE algorithm never exceeded 1500. This shows that the method adopted by the MPE algorithm has a significant effect in suppressing MTTR.

Figure 10 shows the comparison of ATTR of algorithms in the asymmetric scenario. The ATTR of MPE is the shortest in the experiment process. The comparison results show that $\mathrm{ACH}, \mathrm{AHW}$, and SSB with few communication loads (fewer global channels) all have better continuous rendezvous capabilities. It is worth noting that the increase in the number of global channels did increase the differentiation of continuous rendezvous capabilities of all the algorithms. The ATTR of the four algorithms (MPE, ACH, AHW, and SSB) is much smaller than that of the JS algorithm and the RW algorithm, and it is $83.3 \%$ lower than that of the RW algorithm on average. In the comparison of four algorithms (MPE, ACH, AHW, and SSB), MPE also achieves the shortest ATTR, which is up to $53.71 \%$ and $28.88 \%$ lower than the ACH and SSB algorithms. This indicates that the MPE algorithm can also maintain the stability of the rendezvous under asymmetric conditions. 


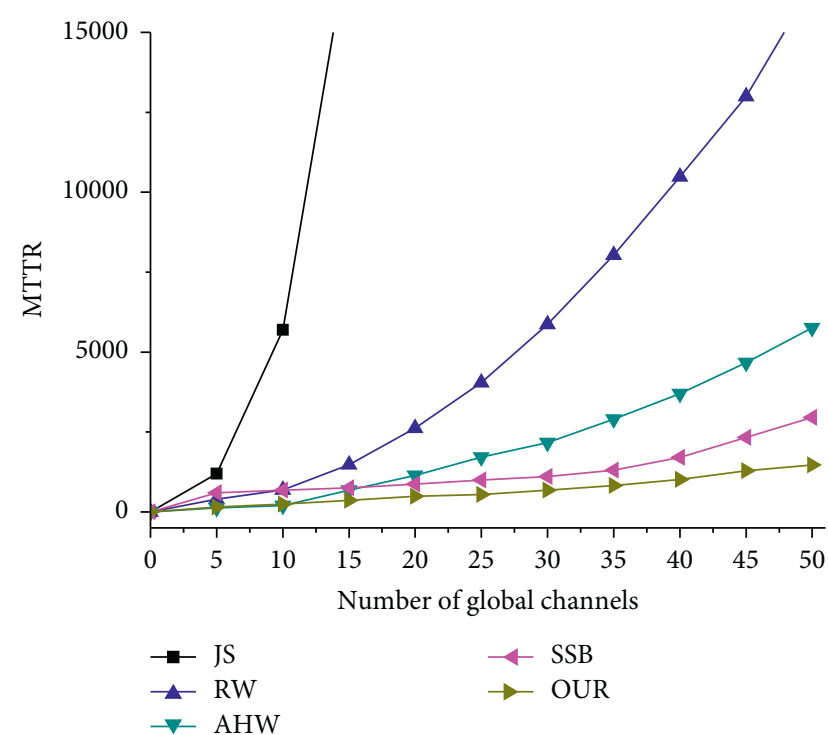

FIgURE 9: MTTR in an asymmetrical scenario.

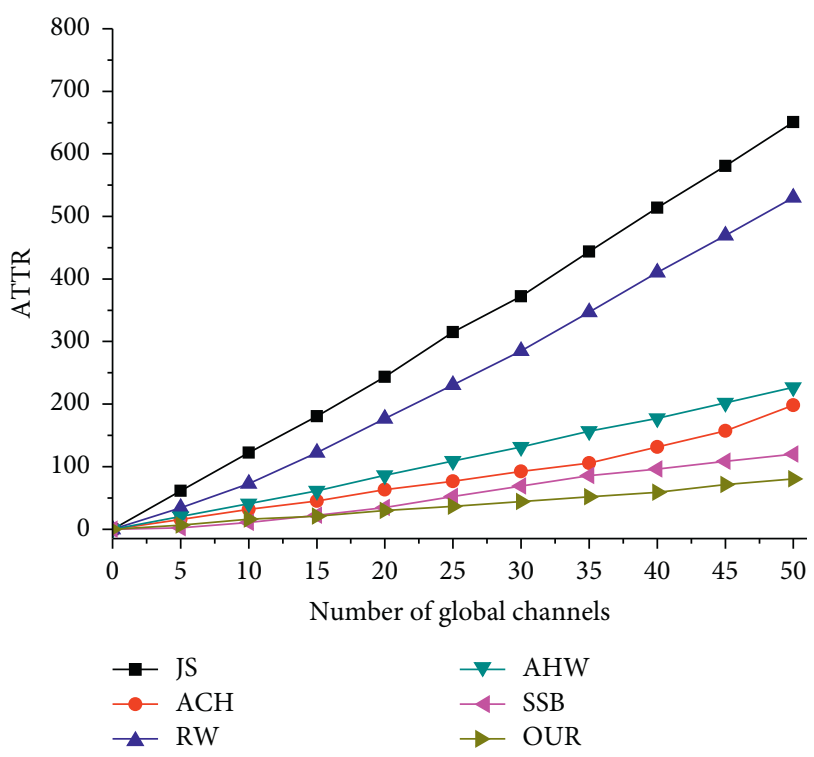

FIgURe 10: ATTR in an asymmetrical scenario.

\section{Conclusions}

In this work, asymmetric and heterogeneous scenarios are the two most determinative factors in the speed of rendezvous. We propose the MPE algorithm to address the above problems. Therefore, MPE can efficiently meet the rendezvous requirements of wireless devices in symmetric and asymmetric scenarios under the heterogeneous channel, resulting in that wireless devices can achieve rendezvous faster and save channel search energy. To evaluate the applicability of MPE, some suitable analytical methods, namely, MTTR and ATTR are also proposed. The results show that the performance of MPE is better than classical blind rendezvous algorithms, namely $\mathrm{ACH}$ and JS.

MPE discusses the rendezvous algorithm in heterogeneous and asymmetric cases only for the two wireless devices. It is not yet able to perform fast rendezvous in multi wireless devices. Therefore, proposing a blind rendezvous method for multiwireless devices in data interaction would be an important future direction for research.

\section{Data Availability}

The data used to support the findings of this study are available from the author upon request.

\section{Ethical Approval}

All applicable international, national, and/or institutional guidelines for the care and use of animals were followed.

\section{Conflicts of Interest}

The authors declare that they have no conflicts of interest in this work.

\section{Acknowledgments}

This work was financially supported by the Scientific Research Project of National Natural Science Foundation of China (no. U1709212) and Zhejiang Province Public Welfare Project of China (no. LGF18F030005).

\section{References}

[1] K. Zhang, J. Ni, K. Yang, X. Liang, J. Ren, and X. S. Shen, "Security and privacy in smart city applications: challenges and solutions," IEEE Communications Magazine, vol. 55, no. 1, pp. 122-129, 2017.

[2] C. Dai, X. Liu, J. Lai, P. Li, and H.-C. Chao, "Human behavior deep recognition architecture for smart city applications in the 5G environment," IEEE Network, vol. 33, no. 5, pp. 206-211, 2019.

[3] H. Zheng, S. Wang, and X. Ping, "Study of spinyhead croaker (collichthys lucidus) fat content forecasting model based on electronic nose and non-linear data resolution model," Food Analytical Methods, vol. 12, no. 1, 2019.

[4] C. Shao, H. Zheng, Z. Zhou et al., "Ridgetail white prawn (exopalaemon carinicauda) $\mathrm{K}$ value predicting method by using electronic nose combined with non-linear data analysis model," Food Analytical Methods, vol. 11, no. 11, pp. 3121-3129, 2018.

[5] J. Peng, L. Zheng, and J. Li, "Sucrose quantitative and qualitative analysis from tastant mixtures based on $\mathrm{Cu}$ foam electrode and stochastic resonance," Food Chemistry, vol. 197, p. 1168, 2015.

[6] H. Yang, Y. Ye, and X. Chu, "Energy efficiency maximization for UAV-enabled hybrid backscatter-harvest-then-transmit communications," IEEE Transactions on Wireless Communications, vol. 99, 2021.

[7] Z. Zhou, C. Shao, and H. Zheng, "Simulating study on RHCRP protocol in utility tunnel WSN," Wireless Networks, vol. 26, no. 99, 2020.

[8] C. Alcaraz, J. Lopez, R. Roman, and H.-H. Chen, "Selecting key management schemes for WSN applications," Computers \& Security, vol. 31, no. 8, pp. 956-966, 2012.

[9] S. Kumar, A. Kumar, and R. K. Vishwkarma, "A survey on routing protocol for wireless sensor network," International 
Journal of Advanced Research in Computer Engineering \& Technology, vol. 2, no. 2, 2013.

[10] O. Olayinka and A. Attahiru, "A survey on an energy-efficient and energy-balanced routing protocol for wireless sensor networks," Sensors, vol. 17, no. 5, p. 1084, 2017.

[11] F. Losilla, A. J. Garcia-Sanchez, and F. Garcia-Sanchez, "A comprehensive approach to WSN-based its applications: a survey," Sensors, vol. 11, no. 11, 2012.

[12] W. Fang, J. Wu, and Y. Bai, "Quantitative risk assessment of a natural gas pipeline in an underground utility tunnel," Process Safety Progress, vol. 38, 2019.

[13] O. G. Zabaleta, J. P. Barrangú, and C. M. Arizmendi, "Quantum game application to spectrum scarcity problems," Physica A: Statistical Mechanics and Its Applications, vol. 466, pp. 455-461, 2017.

[14] H. Zhang, N. Xu, and F. Xu, "Graph cut based clustering for cognitive radio ad hoc networks without common control channels," Wireless Networks, vol. 24, 2016.

[15] T. Y. Lin, K. R. Wu, and G. C. Yin, "Channel-hopping scheme and channel-diverse routing in static multi-radio multi-hop wireless networks," IEEE Transactions on Computers, vol. 64, no. 1, pp. 71-86, 2014

[16] S. Mohapatra and P. K. Sahoo, "ASCH: A novel asymmetric synchronous channel hopping algorithm for Cognitive Radio Networks," in Proceedings of the IEEE International Conference on Communications, IEEE, Kuala Lumpur, Malaysia, May 2016.

[17] Q. Liu, X. Wang, B. Han, X. Wang, and X. Zhou, "Access delay of cognitive radio networks based on asynchronous channel-hopping rendezvous and CSMA/CA MAC," IEEE Transactions on Vehicular Technology, vol. 64, no. 3, pp. 1105-1119, 2015.

[18] Y. T. Wang, G. C. Yang, and S. H. Huang, "Multi-MTTR asynchronous-asymmetric channel-hopping sequences for scalable cognitive radio networks," IEEE Transactions on Cognitive Communications and Networking, vol. 99, p. 1, 2018.

[19] T.-H. Lin, G.-C. Yang, and W. C. Kwong, "A homogeneous multi-radio rendezvous algorithm for cognitive radio networks," IEEE Communications Letters, vol. 23, no. 4, pp. 736-739, 2019.

[20] C. Chang, C. Chen, and D. S. Lee, "Efficient encoding of user IDs for nearly optimal expected time-to-rendezvous in heterogeneous cognitive radio networks," IEEE/ACM Transactions on Networking, vol. 23, 2017.

[21] N. C. Theis, R. W. Thomas, and L. A. Dasilva, "Rendezvous for cognitive radios," IEEE Transactions on Mobile Computing, vol. 10, no. 2, pp. 216-227, 2010.

[22] J. Shin, D. Yang, and C. Kim, "a channel rendezvous scheme for cognitive radio networks," IEEE Communications Letters, vol. 14, no. 10, pp. 954-956, 2010.

[23] Y. Deng, Z. Zhou, Z. Zhao et al., "Simulation study on ASCMP protocol in utility tunnel WSN," IEEE Access, vol. 7, pp. 168141-168150, 2019.

[24] H. Qian, Z. Zong, C. Wu, J. Li, and L. Gan, "Numerical study on the behavior of utility tunnel subjected to ground surface explosion," Thin-Walled Structures, vol. 161, Article ID 107422, 2021.

[25] H. Liu, Z. Lin, X. Chu, and Y.-W. Leung, "Jump-stay rendezvous algorithm for cognitive radio networks," IEEE Transactions on Parallel and Distributed Systems, vol. 23, no. 10, pp. 1867-1881, 2012.

[26] K. Bian, "Maximizing rendezvous diversity in rendezvous protocols for decentralized cognitive radio networks," IEEE
Transactions on Mobile Computing, vol. 12, no. 7, pp. 1294-1307, 2012.

[27] H. Liu, Z. Lin, and X. Chu, "Ring-walk based channel-hopping algorithms with guaranteed rendezvous for cognitive radio networks," in Proceedings of the 2010 IEEE/ACM Int'l Conference on Green Computing and Communications \& Int'l Conference on Cyber, Physical and Social Computing, pp. 755-760, IEEE, Washington, DC, USA, December 2010.

[28] V. A. Reguera, E. O. Guerra, and R. D. Souza, "Short channel hopping sequence approach to rendezvous for cognitive networks," IEEE Communications Letters, vol. 18, no. 2, pp. 289-292, 2013. 